2007-8

\title{
Experiments in Cooperative Timing for Miniature Air Vehicles
}

Derek R. Nelson

Northrop Grumman

Timothy W. McLain

Brigham Young University - Provo, mclain@byu.edu

Randal W. Beard

Brigham Young University - Provo, beard@byu.edu

Follow this and additional works at: https://scholarsarchive.byu.edu/facpub

Part of the Electrical and Computer Engineering Commons, and the Mechanical Engineering

Commons

\section{Original Publication Citation}

Derek R. Nelson, Timothy W. McLain, and Randal W. Beard. "Experiments in Cooperative Timing for Miniature Air Vehicles", Journal of Aerospace Computing, Information, and Communication, Vol. 4, No. 8 (2007), pp. 956-967. doi: 10.2514/1.19422

\section{BYU ScholarsArchive Citation}

Nelson, Derek R.; McLain, Timothy W.; and Beard, Randal W., "Experiments in Cooperative Timing for Miniature Air Vehicles" (2007). Faculty Publications. 1524.

https://scholarsarchive.byu.edu/facpub/1524

This Peer-Reviewed Article is brought to you for free and open access by BYU ScholarsArchive. It has been accepted for inclusion in Faculty Publications by an authorized administrator of BYU ScholarsArchive. For more information, please contact ellen_amatangelo@byu.edu. 


\title{
Experiments in Cooperative Timing for Miniature Air Vehicles
}

\author{
Derek R. Nelson $^{1} \quad$ Timothy W. McLain ${ }^{2} \quad$ Randal W. Beard ${ }^{3}$ \\ ${ }^{1}$ Unmanned Systems Division, Northrop Grumman Corporation, San Diego, California 92127 \\ ${ }^{2}$ Department of Mechanical Engineering, Brigham Young University, Provo, Utah 84602 \\ ${ }^{3}$ Department of Electrical and Computer Engineering, Brigham Young University, Provo, Utah 84602
}

This paper presents experimental results for two cooperative timing missions carried out using a team of three miniature air vehicles (MAVs). Using a cooperative timing algorithm based on coordination functions and coordination variables, the MAV team executed a series of simultaneous arrival and cooperative fly-by missions. In the presence of significant wind disturbances, the average time difference between the first and last vehicle in the simultaneous arrival experiments was $1.6 \mathrm{~s}$. For the cooperative fly-by experiments, the average timing error between vehicle arrivals was $0.6 \mathrm{~s}$. These results demonstrate the practical feasibility of the cooperative timing approach.

\section{Introduction}

The effectiveness of unmanned aerial vehicles (UAVs) in recent years has increased dramatically. This increase is due to advances in microcontroller, battery, and sensor technologies. As a result, the use of UAVs, primarily in military applications, has also increased. Larger UAVs, such as Global Hawk, Predator, and Hunter, are currently being used for border patrol, surveillance, and even target prosecution. Smaller microcontollers and sensor packages have also improved the capabilities of miniature aerial vehicles ${ }^{\mathrm{a}}$, and significant resources have been allocated toward the further development of these technologies. ${ }^{1}$ MAVs have a number of applications for which they are especially well suited because of their size and cost. Some of these applications include reconnaissance, battle damage assessment, and surveillance. Although there are many uses for MAVs in commercial or civilian applications, their reliability and capabilities will have to be improved in order to fully transition into the commercial sector.

\footnotetext{
${ }^{a}$ We consider miniature aerial vehicles to be those with wingspans in the $0.3 \mathrm{~m}$ to $2 \mathrm{~m}$ range and micro aerial vehicles to be those with wingspans under $0.3 \mathrm{~m}$. Here the abbreviation MAV denotes miniature aerial vehicle.
} 
In recent years, cooperative control for teams of UAVs has been a heavily researched area. Chandler, et al. have explored strategies for dealing with the complexity and coupling inherent in cooperative autonomous UAV teams. ${ }^{4}$ They show, by computer simulation, that using network flow and auction algorithms for task assignment yields improved team performance for search missions with varying numbers of team members and tasks to be accomplished.

Much work has also been devoted to path planning for multi-agent teams in high-risk environments. Flint, et al. have developed a model for task assignment and path planning involving both a priori and dynamic information about threats and targets. ${ }^{5}$ Simulation results in a threedimensional environment illustrate the effectiveness of this method. Maza and Ollero present a method for cooperatively searching an area with a heterogeneous team of UAVs taking advantage of the capabilities of the individual agents in the team. ${ }^{6}$ The area to be searched is divided into a set of polygons and the assignments for the searching of these polygons is based on which of the UAVs is best suited for the task. Vincent and Rubin have proposed to use swarming methods for cooperatively searching an area. ${ }^{11}$ The UAVs fly in a formation that utilizes the sensing capabilities of each team member and easily adapts if any of the members is lost during the mission.

This paper extends work presented previously in the area of cooperative timing missions utilizing coordination variables and functions to make path planning and timing decisions. ${ }^{7,8}$ Coordinated timing over a known target can be advantageous in a number of scenarios. One example is the case where a persistent image of a target from a consistent perspective is desired. If the camera footprint for each MAV in a team is known, arrival intervals for the team can be determined such that a persistent image of the target can be obtained. Another scenario is surveillance of a target in a risky environment. A simultaneous arrival of a team of MAVs over the target could be coordinated, thus maximizing the possibility for the team, or at least part of the team, to reach the target. An optimization algorithm based on minimizing fuel consumption was executed to determine paths and velocities for each of the MAVs.

Section II outlines these two scenarios and describes the issues involved in achieving cooperation for a team of MAVs. Section III discusses the technical approach taken to address these issues, and Section IV describes of the experimental setup used. The experimental results in Section V are the primary contribution of this paper and illustrate the feasibility of real-time cooperative timing missions for MAVs.

\section{Problem Overview}

The focus of this paper is experimental demonstration of cooperative timing scenarios for MAVs, thereby providing practical validation of our cooperative control approach. Two specific missions are considered: simultaneous arrival and cooperative fly-by.

For the simultaneous arrival mission, the objective is for the MAVs composing the team to 
arrive at the target at the same time from different approach directions. The ability to have multiple vehicles arriving at destination simultaneously is useful for a variety of tactical purposes including formation join-up and target attack. In an attacking situation, simultaneous arrival maximizes the element of surprise and increases the intensity of the attack.

For the cooperative fly-by mission, the objective is for the MAVs to approach the target along the same approach path at specified timing intervals. The ability to have multiple vehicles fly over a target at specified intervals is useful for persistent surveillance or for prosecuting targets where a sequence of tasks is required (e.g., identification, attack, battle damage assessment). We consider the scenario where the heading through the target is predetermined to be along the imaging path so that a persistent image of the target from the same viewing angle can be obtained.

Both missions have several common elements in their planning and execution. During the initial phase of the mission, the MAVs are occupied with a secondary task (i.e. monitoring secondary targets, positioning themselves for the cooperative mission, etc.). Upon command from the operator at the ground station, paths are planned to satisfy the timing constraints for the mission and to minimize the cooperation objective (e.g., minimize fuel cost). Paths are planned based on current MAV and target locations and the desired headings through the target. Upon completion of the pass over the target, the MAVs return to the secondary tasks they were occupied with during the initial phase of the mission. Challenges associated with these missions include real-time cooperative path planning, reliable and timely communication of cooperation information, and accurate path following with significant wind disturbances.

The cooperative timing solutions for the two missions that we will present were obtained from a cooperative timing algorithm that employed coordination variables and coordination functions to represent critical timing information for the MAVs. The experimental results that will be presented involve a team of three MAVs although the approach applies generally to two or more vehicles. This cooperative timing strategy is outlined in the following section together with the path planning and path following approaches that were used.

\section{Technical Approach}

\section{Cooperative Timing Strategy}

We employ a cooperative timing strategy that is based on the utilization of coordination variables and coordination functions. The underlying concept of coordination variables and coordination functions is that the minimal information essential to achieving a cooperation objective should be identified and communicated among agents on the team. Minimizing the amount of information being sent improves communication speed and reliability. It also tends to simplify cooperative control calculations by reducing the problem to its essential elements.

Let $\mathbf{x}_{i}$ define the situation state for the $i^{\text {th }}$ vehicle on a team. For the cooperation problems 
considered in this paper, the situation state includes information about the current MAV position, the target position, the desired heading through the target, and the time interval between MAV arrivals (zero for the simultaneous arrival scenario). Additional information about the environment (e.g., wind speed and direction) can also be included if it is available. For a given situation $\mathbf{x}_{i}$, the set of feasible decisions for the $i^{\text {th }}$ vehicle is represented by $\mathcal{U}_{i}\left(\mathbf{x}_{i}\right)$, and $\mathbf{u}_{i} \in \mathcal{U}_{i}$ is the decision variable for the $i^{\text {th }}$ vehicle. The choice of the decision variable by each vehicle on the team affects both the existence and the value of the cooperation objective. In the cooperative timing scenarios considered, the decision variable consists of a waypoint path and an average velocity.

The process of cooperation among agents can be viewed as having objectives and constraints. For the simultaneous arrival scenario, the cooperation constraint requires the MAVs to arrive over the target at the same time. For the fly-by scenario, the cooperation constraint requires the MAVs to fly over the target with the second vehicle at a specified time interval behind the first, and the third vehicle arriving at a specified time interval after the second. Cooperation is said to occur if the cooperation constraints are met. In the these scenarios, the cooperation objective is to minimize the battery energy required to complete the mission. The contribution of a vehicle to the team cooperation objective is represented by an influence function, $\phi_{i}=J_{i}\left(\mathbf{u}_{i}\right)$. In this case the energy consumption for the $i^{\text {th }}$ vehicle (represented by $\phi_{i}$ ) is a function of the MAV velocity and the waypoint path (represented by $\mathbf{u}_{i}$ ).

The coordination variable $\theta$ represents the minimal amount information necessary to achieve cooperation. For the simultaneous arrival problem, the coordination variable is the arrival time over the target. For the fly-by problem, the coordination variable is the arrival time of the first MAV and the arrival order for the vehicles. If every vehicle has knowledge of the value of the coordination variable and responds accordingly, cooperative behavior will be achieved by the team. For a vehicle, the value of the coordination variable is related to the decision variable, $\theta_{i}=f_{i}\left(\mathbf{u}_{i}\right)$, and defines what the vehicle can do to ensure that cooperation constraints are met. In the scenarios considered in this paper, the choice of path and velocity determines MAV energy consumption and arrival time and therefore influences both the cooperation objective and the cooperation constraint. Under the assumptions that battery power is proportional to aerodynamic drag and that the MAV flies at constant speed, the battery energy consumption is given by

$$
J_{i}=c_{b} v_{i} L_{i}
$$

where $c_{b}>0$ is a constant, $v_{i}$ is the MAV velocity, and $L_{i}$ is the length of the waypoint path.

For the cooperative fly-by scenario with three MAVs, the cooperation constraints can be written 


$$
\begin{aligned}
& T_{1}=T_{s}, \\
& T_{2}=T_{s}+\Delta_{2}, \\
& T_{3}=T_{s}+\Delta_{3},
\end{aligned}
$$

where $T_{i}$ is the arrival time of the $i^{\text {th }}$ vehicle, $\Delta_{i}$ represents the interval between the arrival of the first and $i^{\text {th }}$ vehicles, and $\theta=T_{s}$ is the coordination variable. Note that for the simultaneous arrival scenario $\Delta_{2}=\Delta_{3}=0$.

Critical to the implementation of this approach is the definition of the coordination function. The coordination function models a vehicle's influence on the cooperation objective in terms of what the agent can do to meet the cooperation constraints. The coordination function, $\phi_{i}\left(\theta_{i}\right)$ is derived from the influence function and coordination variable as

$$
\begin{aligned}
\phi_{i} & =J_{i}\left(\mathbf{u}_{i}\right) \\
& =J_{i}\left[f_{i}^{\dagger}\left(\theta_{i}\right)\right] \\
& =\phi_{i}\left(\theta_{i}\right),
\end{aligned}
$$

where $f_{i}^{\dagger}$ is the pseudoinverse of $f_{i}$. Typically, $f_{i}$ is not a one-to-one mapping in that numerous values of the decision variable (e.g., waypoint paths, velocities) can result in a single value of the coordination variable (e.g., time over target). The pseudoinverse of $f_{i}$ is found by taking the value of decision variable that minimizes the influence function $J_{i}$, thus creating a one-to-one map between $\theta_{i}$ and $\mathbf{u}$. In other words, when multiple options exist to get the same result, the lowest-cost option is chosen.

Using coordination variables and coordination functions, the battery energy required to complete the mission can be minimized by solving the optimization problem

$$
\theta^{*}=\arg \min _{\theta}\left[\phi_{1}(\theta)+\phi_{2}\left(\theta+\Delta_{2}\right)+\phi_{3}\left(\theta+\Delta_{3}\right)\right]
$$

which implies that the constraint in Equation (1) is satisfied. Once a team optimal value for the coordination variable is found, vehicle decisions can be found from the relationship

$$
\mathbf{u}_{i}=f_{i}^{\dagger}\left(\theta^{*}\right)
$$

Figure 1 illustrates the coordination functions calculated for a cooperative timing mission based on input information provided to the algorithm. Because the coordination function for each UAV is monotonically decreasing, the team-optimal arrival time for one of the vehicles will always lie at the right extreme of its coordination function. For the simultaneous arrival scenario, the solution 
is simply determined by finding the largest arrival time that all three MAVs have in common. For the fly-by scenario, determining the minimum-energy arrival time and order for the team involves a simple search through the right extreme (minimum) values of each coordination function with an evaluation of the team objective to determine the best values. ${ }^{8}$

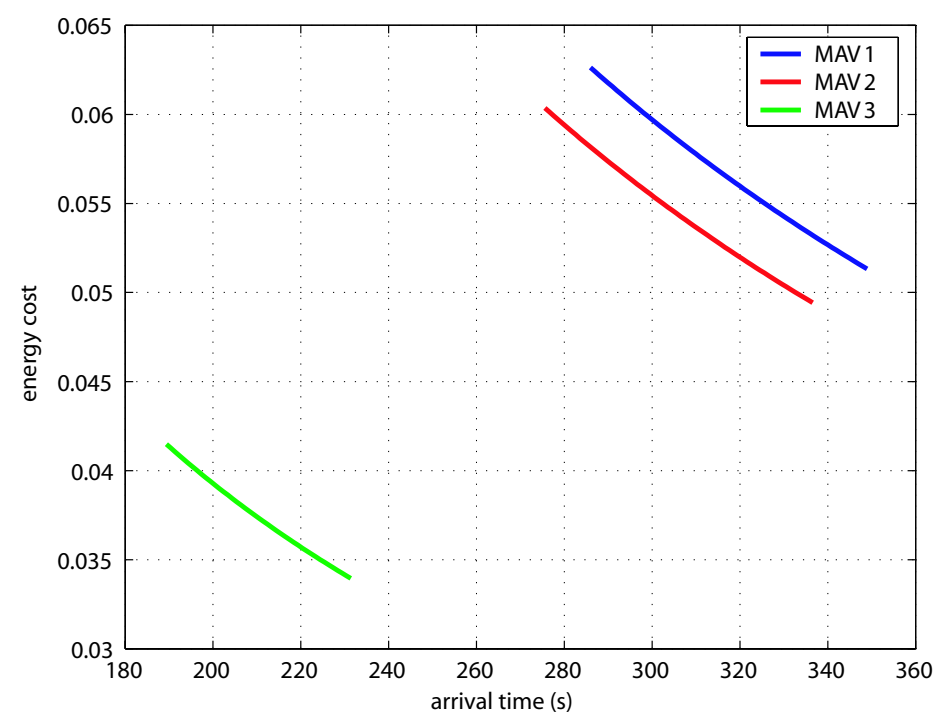

Figure 1. Cooperative timing coordination functions.

\section{Path Planning}

In order to simplify the path planning for this initial implementation, some assumptions about the environment were made. The first assumption was that there were no threats to be avoided. The next assumption was that both the target location and the desired fly-by heading were known, which eliminated any initial target observation and detection steps prior to the cooperative timing mission. Because there were no threats to avoid, a straightforward heuristic approach for planning straight-line waypoint paths was utilized. Straight-line paths were easy to plan and simplified both the distance calculations and timing optimization.

Figure 2 shows an example of the waypoint paths used in the experiments. Waypoint 1 for each agent was calculated to be $100 \mathrm{~m}$ along the same heading that the agent had when the coordination algorithm was executed. Waypoint 2 was set to be a fixed distance from the known target location along the desired approach path. For the experiments, this distance was between $200 \mathrm{~m}$ and $300 \mathrm{~m}$. Allowing this much distance ensured that the transient path following errors associated with transitioning from one straight line path to another would be damped out and that the MAVs would be flying along the desired approach heading when they passed over the target. Waypoint 3 was the target location. Waypoint 4 was collinear with waypoints 2 and 3 on the opposite side of the target from waypoint 2 . Waypoints 2, 3, and 4 were the same for each agent in the fly-by scenario and 


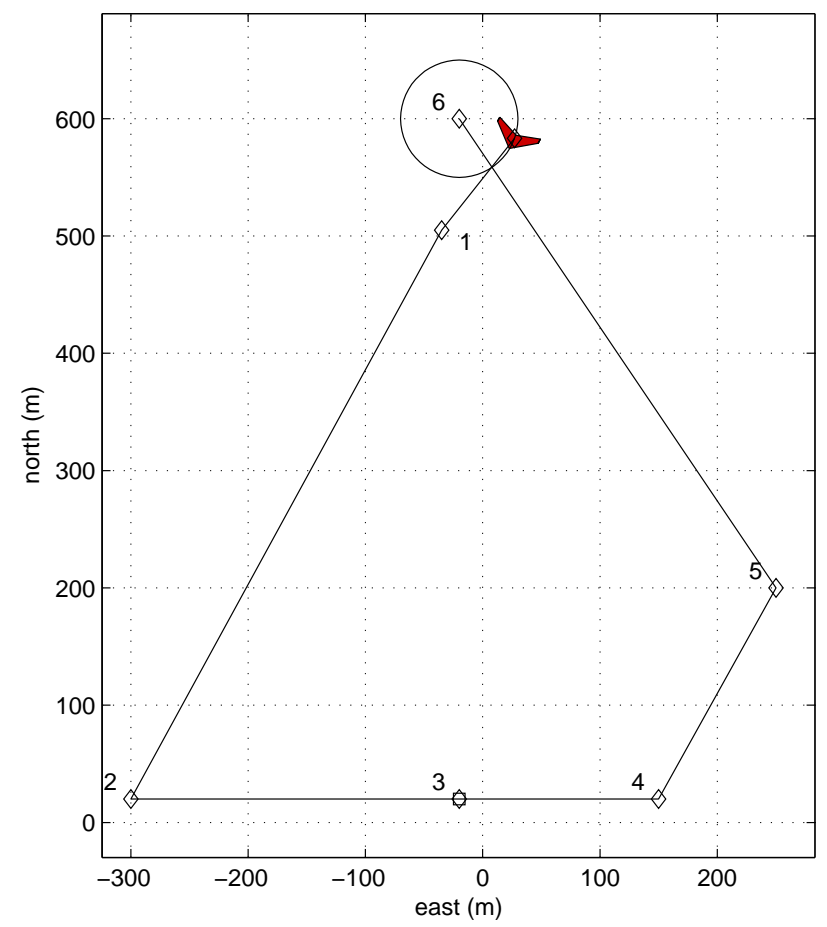

Figure 2. Waypoint path example.

only waypoint 3 was the same in the simultaneous arrival scenario. These three points are referred to as fly-through points. Waypoint 5 was a waypoint to help the agents distance themselves from the target before returning to their final loiter positions, waypoint 6 .

In some cases, depending on the initial MAV positions and headings, the coordination functions do not yield a solution that satisfies the cooperative timing constraint. For example, a cooperative fly-by with a spacing interval of $10 \mathrm{~s}$ between vehicles could not be accomplished with the coordination functions of Figure 1 due to the time gap between the coordination function of UAV 1 and those of UAVs 2 and 3. In these instances, it became necessary to develop some simple approaches for adding length to one or more paths so that the timing constraints could be met.

Based on the coordination functions, the length to be added to a path was calculated to ensure constraint feasibility. If the length that needed to be added was less than $150 \mathrm{~m}$, the location of the first waypoint was adjusted using the law of cosines so that the total distance would be the original length plus the necessary additional length. If the additional length was longer than $150 \mathrm{~m}$, two additional waypoints were added to the path immediately following the first waypoint. The second waypoint was calculated so that its distance from the first waypoint was half of the required additional distance, and the waypoint was placed in a direction directly opposite the target. The third waypoint was placed at the same location as the first, thus effectively adding the required length to the path. The same path shown in Figure 2 with these added points is shown in Figure 3.

Figure 4 shows how the coordination function for UAV 3 was modified by adding length to the 


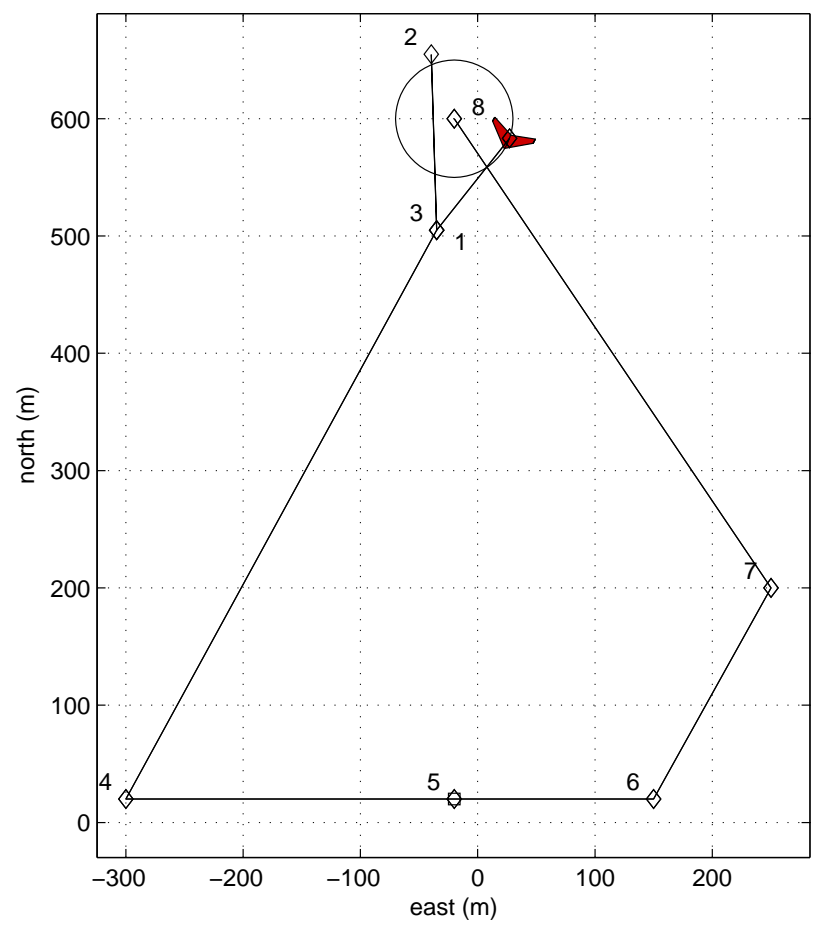

Figure 3. Waypoint path example with added length.

original path. With the added length, the coordination function for UAV 3 shifted up and to the right allowing the $10 \mathrm{~s}$ interval timing constraint to be satisfied.

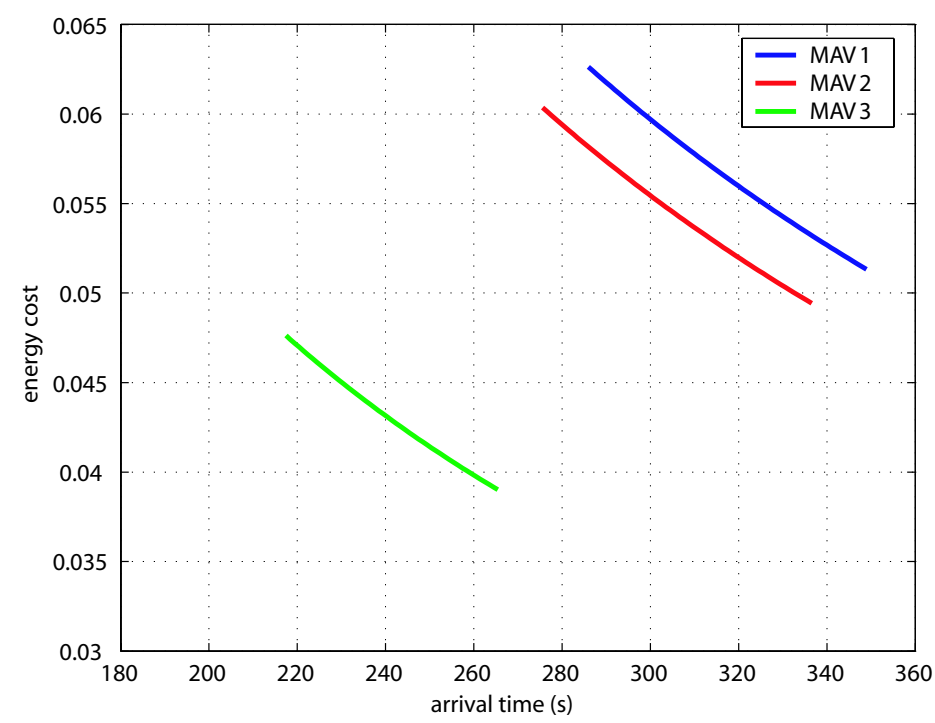

Figure 4. Cooperative timing coordination functions with length added for UAV 1. 


\section{Path Following and Velocity Control}

For timing missions, preserving path length is critical in order to be able to satisfy the timing constraints. Flying extra distance due to poor path following adds unwanted time to the mission. To address this issue, a vector field path following method has been implemented to enable the MAVs to accurately follow the planned waypoint paths. It has been shown in Refs. 9 and 10 that creating vector fields of desired heading in order to direct the MAV onto the path will result in asymptotically decaying tracking error provided ground track heading and ground speed are used in the control law. Use of this method decreased path following error, and consequently, overall path length error.

Since wind speed is typically 10 to 50 percent of the airspeed for a MAV, wind can have adverse effects on path following and timing constraints. Typically, throttle is controlled based on a desired airspeed. This creates problems when trying to fly timing missions in the presence of wind. To address this issue, the throttle was controlled based on ground speed with a check to make sure that the airspeed did not drop below the MAV stall speed. Throttle control based on ground speed also helps reduce the influence of path length error on timing.

\section{Experimental Setup}

\section{Hardware Description}

BYU has developed a reliable and robust experimental platform to flight test algorithms for MAVs. ${ }^{2,3}$ Figure 5 shows the key elements of the testbed. The first frame shows BYU's Kestrel autopilot which is equipped with a Rabbit $340029 \mathrm{MHz}$ processor, rate gyros, accelerometers, absolute and differential pressure sensors. The autopilot measures $3.8 \times 5.1 \times 1.9 \mathrm{~cm}$ and weighs $18 \mathrm{~g}$. The second frame in Figure 5 shows the airframes used for the flight tests reported in this paper. The airframe is a $1.1 \mathrm{~m}$ wingspan Unicorn EPP foam flying wing, which was selected for its durability, ease of component installation, and flight characteristics. Embedded in the airframe are the Kestrel autopilot, batteries, a $1000 \mathrm{~mW}, 900 \mathrm{MHz}$ radio modem, a GPS receiver, a video transmitter, and a small analog camera. The third frame in Figure 5 shows the ground station components. A laptop runs the Virtual Cockpit software that interfaces through a communication box to the MAVs. An $\mathrm{RC}$ transmitter is used as a stand-by fail-safe mechanism to facilitate safe operations.

\section{Cooperative Control Algorithm}

The cooperative timing algorithm was implemented as part of the Virtual Cockpit ground station software. The current implementation is centralized, in that the ground station acquired all the information required to do the cooperative timing calculations from the vehicles, performed the calculations, and sent only the paths to be flown and desired arrival times back up to the MAVs. 

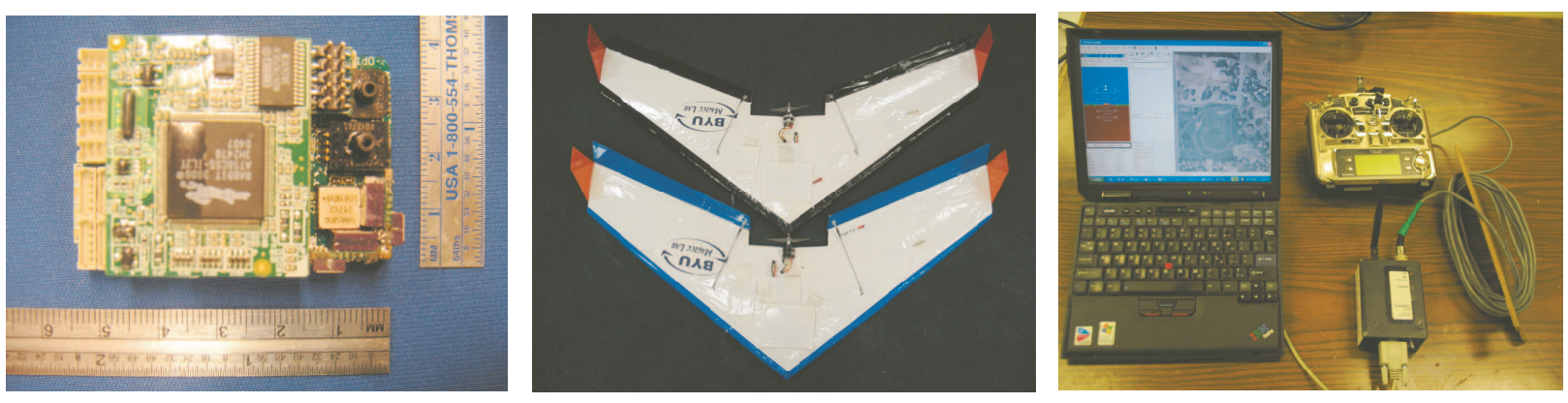

Figure 5. (a) Kestrel autopilot, (b) Unicorn airframes, (c) ground station components.

Execution of the algorithm took approximately $1 \mathrm{~s}$, with a significant portion of the time being consumed by communication between the MAVs and the ground station. Once the MAVs received the paths and arrival times, the autopilot controlled the heading using a vector field $\operatorname{method}^{9}$ and the throttle based on ground speed so that cooperative timing could be achieved. The algorithm used in this paper is shown in Table 1.

Table 1. Cooperative timing algorithm.

\begin{tabular}{ll}
\hline 1 & Acquire GPS locations and headings for each MAV \\
2 & Calculate waypoint paths for each MAV \\
3 & Compute range of arrival times for each MAV \\
4 & Compute coordination function for each MAV \\
5 & Based on coordination functions, choose team optimal coordination \\
& variable (arrival time of first MAV and arrival order for fly-by and \\
& arrival time for simultaneous arrival) \\
6 & Calculate desired arrival time for each MAV \\
7 & Send waypoint paths and desired arrival times to each MAV \\
8 & MAVs fly waypoint paths controlling velocities to achieve cooperation \\
\hline
\end{tabular}

\section{Results and Discussion}

\section{Simultaneous Arrival}

Consecutive simultaneous arrival runs were flown during a 25-minute flight test. Telemetry data from one of the runs is shown in Figure 6. Wind was between 30 and 60 percent of the MAV airspeed during each of the four runs. The average time between the arrival of the first MAV at the target and the arrival of the final MAV was $1.6 \mathrm{~s}$. Range-to-target data is displayed in Figure 7. This illustrates the near simultaneous arrival of the MAVs at the target enabled by the cooperative timing algorithm. Video footage of a simultaneous arrival mission can be viewed by clicking here. 

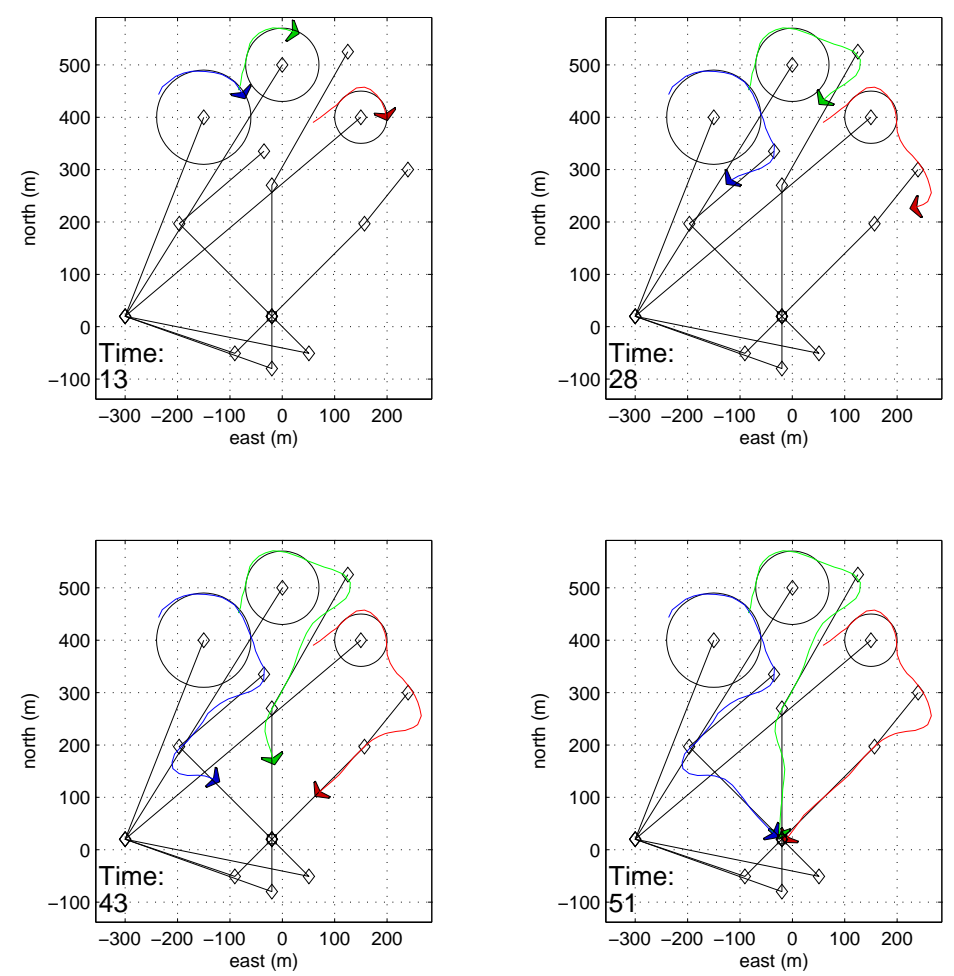

Figure 6. Simultaneous arrival telemetry.

\section{Cooperative Fly-by}

The fly by scenario was flown six times during a half-hour flight involving three MAVs. The wind speeds during the flight were between 30 and 60 percent of the MAV airspeed and were from the southwest. The position telemetry from one of the fly-bys is plotted in Figure 8 . The desired arrival intervals for this run were $3 \mathrm{~s}$ for both intervals and the actual arrival intervals were 3 and 4 s, respectively. The average error in arrival time for the six runs, in spite of the high wind conditions, was approximately $0.6 \mathrm{~s}$. The range to the target versus time for each of the MAVs is shown in Figure 9. The rise in the range curves results from the overshoot that occurs after the first of the fly-through waypoints. This illustrates the arrival of the MAVs at approximately $3 \mathrm{~s}$ intervals. Video footage of a cooperative fly-by mission can be viewed by clicking here.

Figure 10 illustrates a scenario where the length of the path of one of the MAVs had to be lengthened to satisfy the timing constraint. The MAV that began in the bottom of the frame was considerably closer to the target when the fly-by command was issued and its path length had to be increased for cooperation to occur. Two waypoints were added after the first waypoint using the 


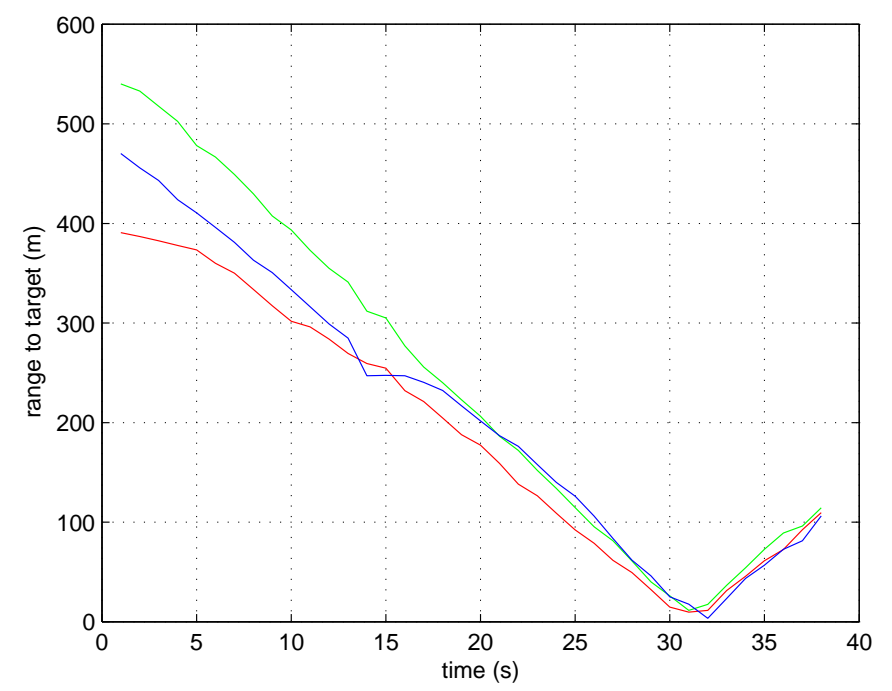

Figure 7. Simultaneous arrival range to target.
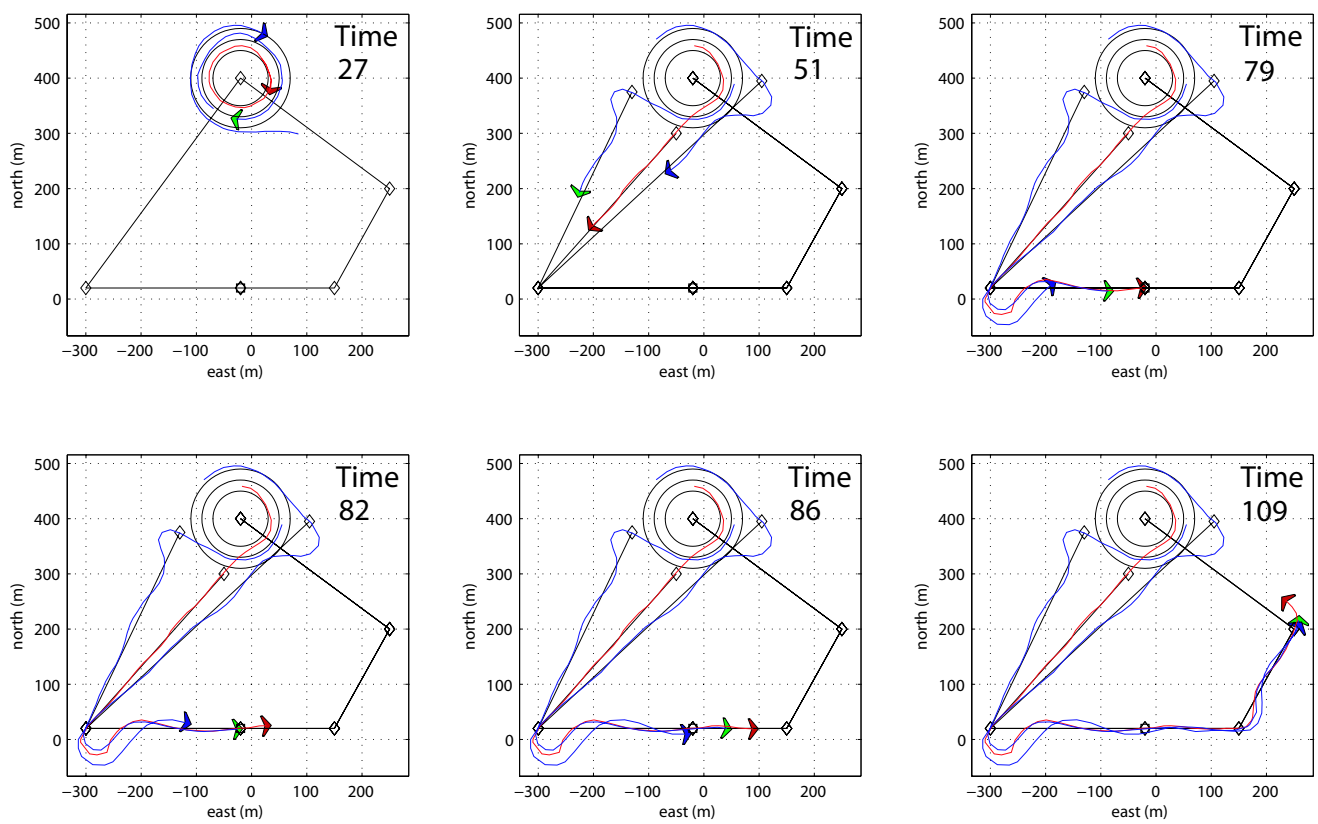

Figure 8. Fly-by telemetry, $3 \mathrm{~s}$ arrival intervals.

method described in Section III. The first two MAVs arrived at the desired arrival times while the third MAV was delayed slightly due to the strong head wind it faced during most of the run.

Although the timing errors for both simultaneous arrival and cooperative fly-by are relatively small, there are a few possible explanations for the errors that occurred. One of the more obvious reasons was the presence of the high relative wind speeds. If the desired groundspeed was in 


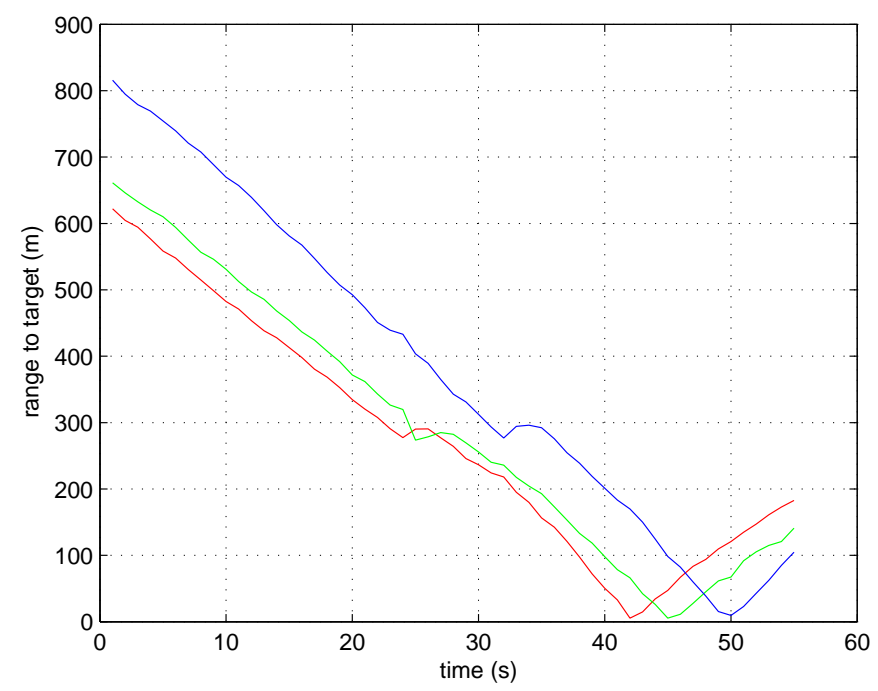

Figure 9. Fly-by range to target, $3 \mathrm{~s}$ arrival intervals.
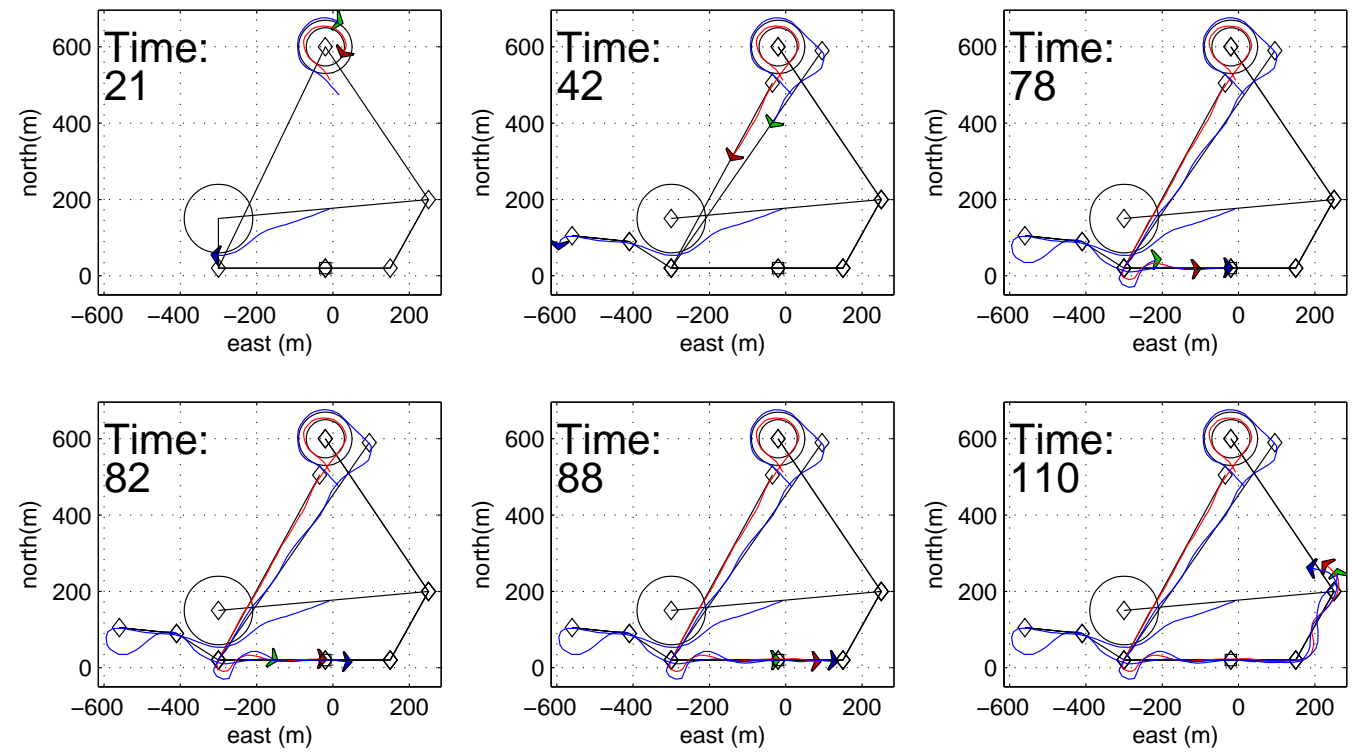

Figure 10. Fly-by telemetry with path lengthened for MAV 1.

the upper end of the feasible airspeed envelope $(20 \mathrm{~m} / \mathrm{s})$ while flying into a headwind, it was difficult for timing constraints to be met. Another issue was synchronizing the timing on each of the autopilots. Each autopilot had its own timer that started when the list of waypoints finished uploading from the ground. This took about $1 \mathrm{~s}$ for each of the three agents and if it took longer for one MAV to finish downloading the information than another, the start times were different. Transient behavior involved in matching the desired groundspeed as it changed and GPS error could also have influenced proper timing. 


\section{Conclusion}

Cooperative timing missions for miniature aerial vehicles have been explored in this paper. Experimental results were obtained using an algorithm based on coordination functions and coordination variables. From these results, we conclude that this coordination algorithm is a viable approach for real-time coordination of teams of multiple MAVs. This conclusion is reinforced by repeatable results from multiple flight test scenarios conducted in high winds.

\section{Acknowledgments}

This research was supported by the Air Force Office of Scientific Research, Award No. FA955004-0209.

\section{References}

${ }^{1}$ Unmanned Aerial Vehicle Roadmap 2002-2007. Technical report, Office of the Secretary, U.S. Department of Defense, December 2002.

${ }^{2}$ R. Beard, D. Kingston, M. Quigley, D. Snyder, R. Christiansen, W. Johnson, T. McLain, and M. Goodrich. Autonomous vehicle technologies for small fixed wing UAVs. AIAA Journal of Aerospace, Computing, Information, and Communication, 2005. (to appear).

${ }^{3}$ R. W. Beard, D. Lee, S. Thakoor, and S. Zornetzer. A new approach to observation of descent and landing of future Mars mission using bioinspired technology innovations. AIAA Journal of Aerospace Computing, Information, and Communication, 2005. (to appear).

${ }^{4}$ P. R. Chandler, M. Pachter, D. Swaroop, J. M. Fowler, J. H. Howlett, S. Rasmussen, C. Schumacher, and K. Nygard. Complexity in UAV cooperative control. In American Control Conference, Anchorage, AK, May 2002.

${ }^{5}$ M. Flint, E. Fernandez-Gaucherand, and M. Polycarpou. Cooperative control for UAVs searching risky environments for targets. In Conference on Decision and Control, Maui, HI, December 2003.

${ }^{6}$ I. Maza and A. Ollero. Multiple UAV cooperative searching operation using polygon area decomposition and efficient coverage algorithms. In 7th International Symposium on Distributed Autonomous Robotic Systems, Toulouse, France, June 2004.

${ }^{7}$ T. W. McLain and R. W. Beard. Unmanned air vehicle testbed for cooperative control experiments. In American Control Conference, pages 5327-5331, Boston, MA, June 2004.

${ }^{8}$ T. W. McLain and R. W. Beard. Coordination variables, coordination functions, and cooperative timing missions. AIAA Journal of Guidance, Control and Dynamics, 28(1):150-161, January 2005.

${ }^{9}$ D. Nelson, T. McLain, and R. Beard. Vector field path following for small unmanned air vehicles. IEEE Transactions on Control Systems Technology, 2005. (submitted).

${ }^{10}$ D. R. Nelson. Cooperative control of miniature air vehicles. Master's thesis, Brigham Young University, Department of Mechanical Engineering, December 2005.

${ }^{11} \mathrm{P}$. Vincent and I. Rubin. A framework and analysis for cooperative search using UAV swarms. In Proceedings of the 2004 ACM symposium on Applied Computing, Nicosia, Cyprus, March 2004. 